\title{
THE CHROMOSPHERIC MAGNETOGRAPH
}

\author{
GLENN J. VEEDER and HAROLD ZIRIN \\ Hale Observatories, Carnegie Institution of Washington, \\ California Institute of Technology, Pasadena, Calif., U.S.A.
}

(Received 11 November, 1969)

\begin{abstract}
By comparison of photoelectric magnetograms with high resolution $\mathrm{H} \alpha$ pictures it is possible to formulate a set of rules by which the magnetic field may be derived directly from the filtergrams. This is possible because of the regularities of magnetic field configurations on the sun and because chromospheric morphology is determined by the magnetic field.

Off-band pictures (preferably $0.5 \AA$ red) show a well-defined enhanced chromospheric network, the boundaries of which coincide with the $5 \mathrm{G}$ contour of longitudinal field on the Mt. Wilson magnetograms. The actual fields are presumably more concentrated along the dark structure of the network. Higher fields are marked by filled-in cells.

Regions of predominantly transverse fields may be inferred from the absence of normal network structure and the presence of chromospheric fibrils. The quiet chromosphere is recognized by the presence of oscillatory motion and the absence of fibrils or strong network structure. Thus, the chromosphere may be divided into three types of regions: enhanced network, horizontal field, and quiet network.

The polarity of the magnetic field may be recognized by plage-antiplage asymmetry; that is, the fact that only following magnetic fields show bright plage in the center of $\mathrm{H} \alpha$.
\end{abstract}

\section{Introduction}

In recent years considerable interest has been devoted to non-photoelectric measurements of solar magnetic fields, which could give a two-dimensional picture of these fields without raster scanning. One such sensor of magnetic fields is the structure of the chromosphere itself, which is strongly governed by the magnetic fields. This has been known since the first magnetograph work and was placed on a solid basis by Howard and Harvey (1964). They showed that the $15 \mathrm{G}$ contour of longitudinal field intensity coincided with the boundaries of calcium emission and that prominences marked the boundary between regions of opposite polarity.

In the present work we describe comparisons of medium resolution $\mathrm{H} \alpha$ pictures and Mt. Wilson magnetograms. Among other things we show:

(1) High quality off-band pictures may be used to draw contours of longitudinal magnetic field strength. The boundary of the network of enhanced, dark spicules in an active region coincides with the $5 \mathrm{G}$ contour of magnetic field. Darker structures inside this contour can be identified with higher fields.

(2) Horizontal (transverse) magnetic fields may be distinguished by fibril structure. There is a region of horizontal field in the disturbed chromosphere between the $5 \mathrm{G}$ contour and the $0 \mathrm{G}$ contour (of longitudinal field). This region surrounds the $5 \mathrm{G}$ contour and is clearly distinguished from the undisturbed chromosphere.

(3) The polarity of the (vertical) magnetic fields can be distinguished by the existence of plages and 'antiplages' in on-band pictures. That is, $\mathrm{H} \alpha$ brightness depends not only on the magnitude of the local magnetic field, but also on its polarity. These 
pictures show that regions of following polarity in any active region are bright for fields above $\sim 30 \mathrm{G}$, but that regions of the same field strength of preceding polarity are not bright. In off-band pictures, the plage is generally darker than the antiplage which in turn is darker than disturbed regions of lower field strength. The plageantiplage difference in the K-line is more subtle; both are bright, but the plage is brighter. Prominences are useful indicators of the neutral lines in the longitudinal field that separate regions of opposite polarity. In the case of emerging bipolar field ('bright regions with loops') the asymmetry does not exist; both preceding and following regions are bright (see Figure 6, arrow No. 1).

The $\mathrm{H} \alpha$ photos used here were taken exclusively with the $12 \mathrm{~cm}$ aperture Caltech photoheliograph located on the roof of the Robinson Astrophysical Laboratory in Pasadena. A $\frac{1}{2} \AA$ Halle filter was used, except in January 1969 when a $\frac{1}{4} \AA$ Zeiss filter was used. The Halle filter is normally tuned by temperature to avoid side bands. The image size is $5 \mathrm{~cm}$.

\section{Identifications of Magnetic Features from $\mathrm{H} \alpha$ Filtergrams}

After comparing many $\mathrm{H} \alpha$ pictures with the Mt. Wilson magnetograms, we have gained enough experience to be able to indicate the regions of opposite polarity and to outline the $5 \mathrm{G}$ contour from a pair of filtergrams (one at $\mathrm{H} \alpha$ and one at $\mathrm{H} \alpha+0.5 \AA$ ) without the corresponding magnetogram. We then compare our predictions with the magnetograms when they become available in order to improve our future predictions. In general, we find better agreement for good quality pictures and for regions that have a simple bipolar structure. Exceptionally good seeing is required to point out regions of fields greater than $5 \mathrm{G}$ with any accuracy. The present method is limited by the quality of existing magnetograph data, which must be used to establish the rules for associating chromospheric structure with magnetic field configurations. As better magnetograph data becomes available, new rules will inevitably be formulated.

Figures 1 and 2 show pictures taken at $\mathrm{H} \alpha$ and $\mathrm{H} \alpha+0.5 \AA$ of an active region on July 17,1968 . This region is predominantly bipolar with one large spot group followed by a number of plages of following polarity. In addition, we have a complicated neutral line that separates the opposite polarities in the bipolar group region from some regions of higher fields to the north.

In order to predict the $\pm 5 \mathrm{G}$ contours, we first outline the region of enhanced chromospheric network on an overlay of the picture taken at $\mathrm{H} \alpha+0.5 \AA$. We then compare this outline with the picture of the region at centerline $\mathrm{H} \alpha$ to help us decide which regions have which polarity and whether we have drawn a contour around a bipolar region that should be split into two contours of opposite polarity.

At this stage it is necessary to make two fairly safe assumptions. In general, a large spot group followed by some bright plages is bipolar and the preceding spot itself has the leading polarity of that solar cycle and hemisphere. This is particularly true if there is no bright plage immediately surrounding the spot. We then assume that this is indeed the case for our particular region. 


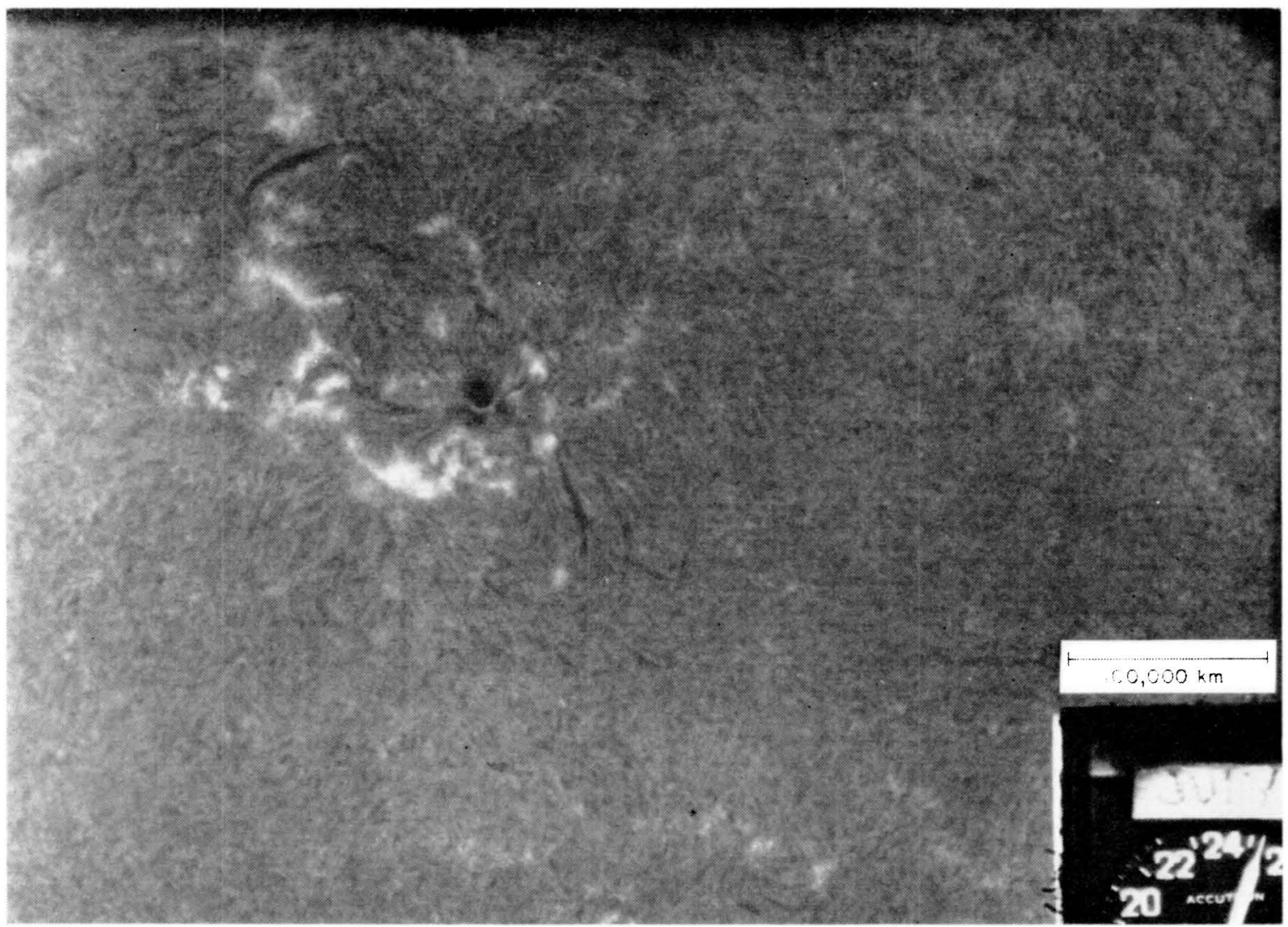

Fig. 1. Bipolar group July 17, $1968 \mathrm{H} \alpha$.

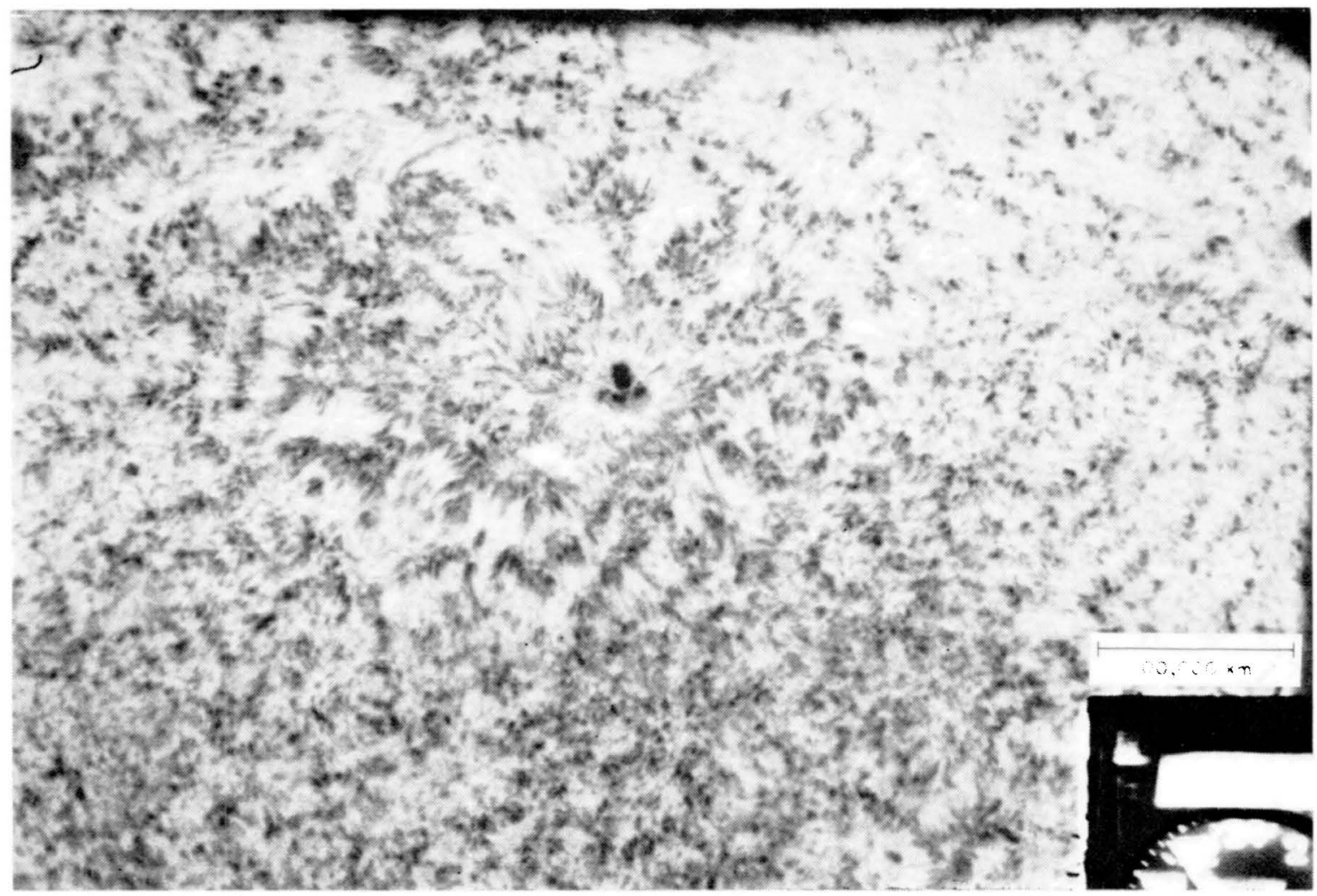

Fig. 2. Bipolar group July $17,1968 \mathrm{H} \alpha+\frac{1}{2} \AA$. 


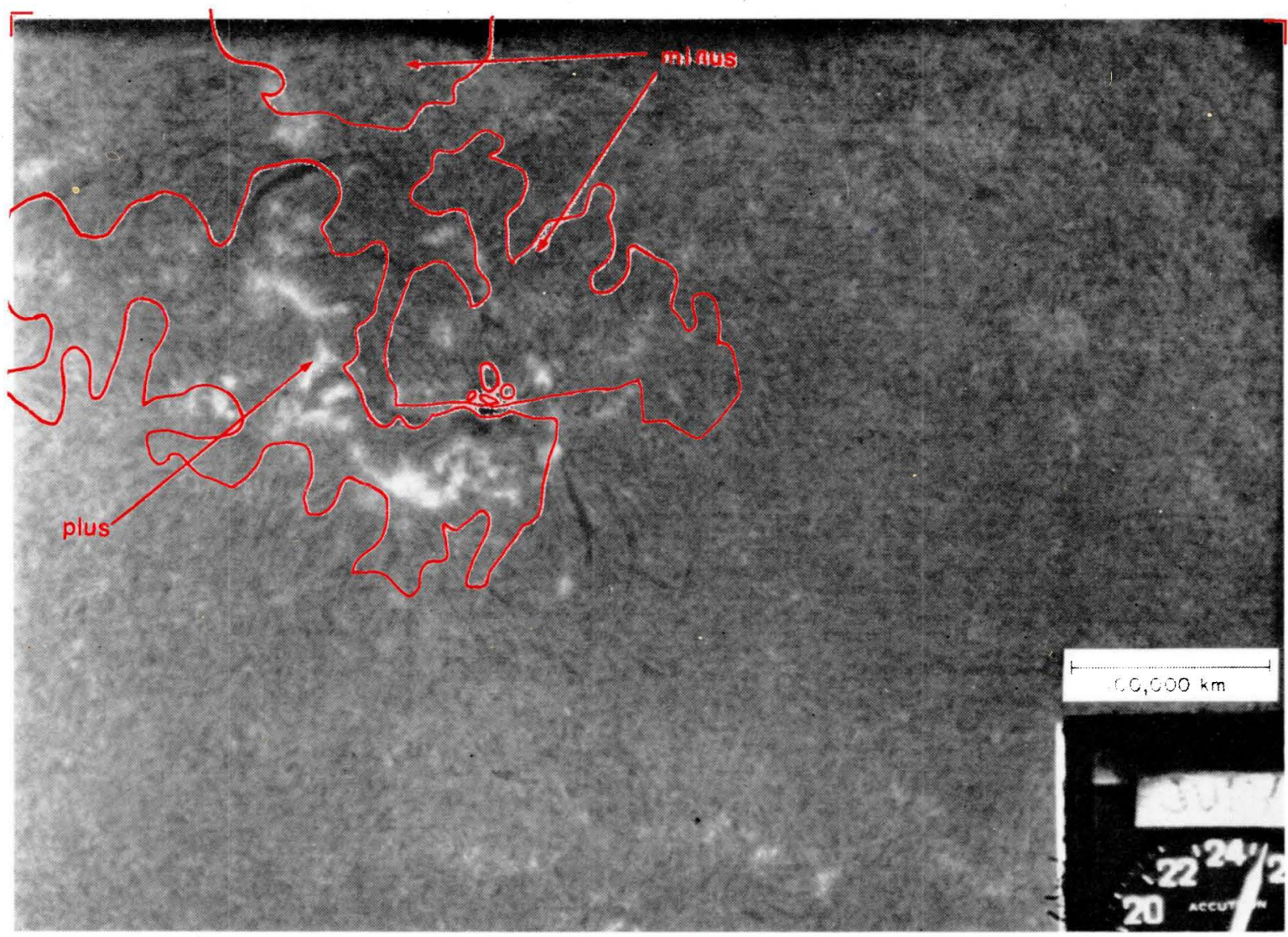

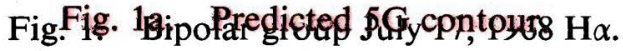

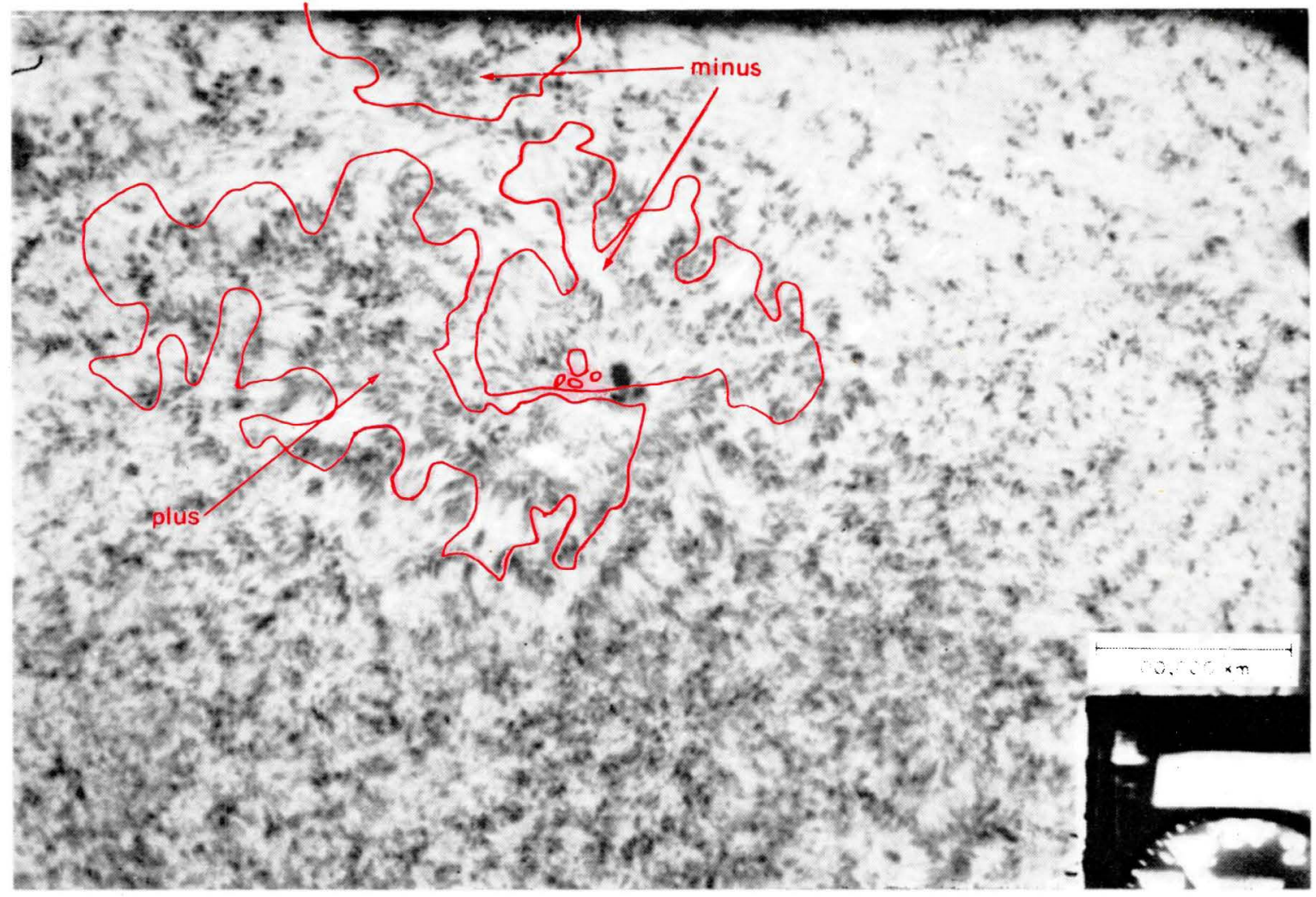

Fig. 2. Bipolar group July $17,1968 \mathrm{H} \alpha+\frac{1}{2} \AA$. 
Prominences on the centerline $\mathrm{H} \alpha$ pictures help indicate the lines of neutral magnetic field (i.e. where the field is horizontal). Regions dominated by horizontal fibrils also mark the regions of zero longitudinal fields. Since prominences usually only occur along limited sections of neutral lines, these fibrils are useful in helping to 'connect' prominences and thus establish the entire neutral line. With the above assumptions and the knowledge that the polarity of the (vertical) magnetic field must change every time we cross such a neutral line, we can assign a polarity to every region we have outlined.

The most difficult neutral line to establish is the one between the spot group and the following plages. The plages seem to disturb the chromosphere so much that the narrow region where the field becomes less than $5 \mathrm{G}$ as it reverses sign is difficult to pick out. However, it must lie between the spot group and the sharp rise of brightness in centerline $\mathrm{H} \alpha$ of the following plages. The principal difficulty in predicting the $5 \mathrm{G}$ contour is the low resolution $\left(17^{\prime \prime}\right)$ of the magnetograms as well as the errors in data reduction which sometimes results in incomplete contours. Isolated strong fields are often lost. Figure 1a is an overlay of the field predicted from these considerations.

Figure 3 is an overlay of the Mt. Wilson magnetogram on our prediction of the field. Our sketch is close to the $5 \mathrm{G}$ contour, but the agreement is not perfect for the

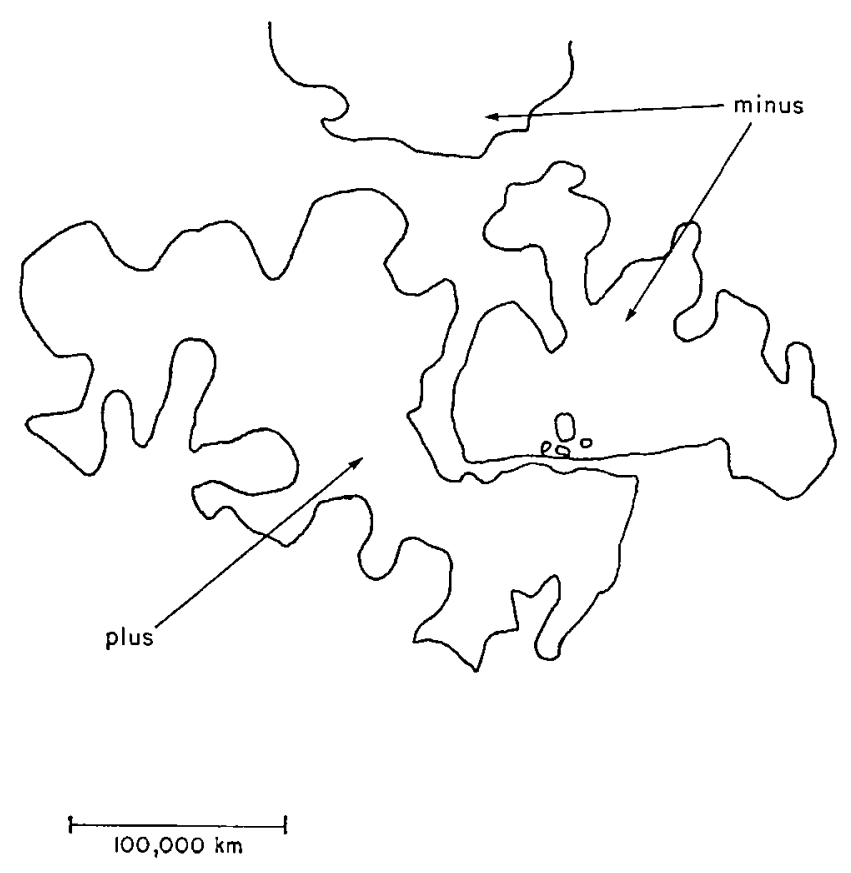

Fig. 3. Predicted 5 G magnetic contour July 17, 1968. 
$\Gamma$ Prominences on the centerline $\mathrm{H} \alpha$ pictures help indicate the lines of neutral magnetil field (i.e. where the field is horizontal). Regions dominated by horizontal fibrils also mark the regions of zero longitudinal fields. Since prominences usually only occur along limited sections of neutral lines, these fibrils are useful in helping to 'connect' prominences and thus establish the entire neutral line. With the above assumptions and the knowledge that the polarity of the (vertical) magnetic field must change every time we cross such a neutral line, we can assign a polarity to every region we have outlined.

The most difficult neutral line to establish is the one between the spot group and the following plages. The plages seem to disturb the chromosphere so much that the narrow region where the field becomes less than $5 \mathrm{G}$ as it reverses sign is difficult to pick out. However, it must lie between the spot group and the sharp rise of brightness in centerline $\mathrm{H} \alpha$ of the following plages. The principal difficulty in predicting the $5 \mathrm{G}$ contour is the low resolution $\left(17^{\prime \prime}\right)$ of the magnetograms as well as the errors in data reduction which sometimes results in incomplete contours. Isolated strong fields are often lost. Figure 1a is an overlay of the field predicted from these considerations.

Figure 3 is an overlay of the Mt. Wilson magnetogram on our prediction of the field. Our sketch is close to the $5 \mathrm{G}$ contour, but the agreement is not perfect for the



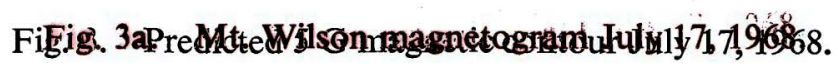


reasons indicated. In general, we have indicated the regions of magnetic fields stronger than $5 \mathrm{G}$ and have assigned the correct polaritiès to them. On a smaller scale, our contour tends to include somewhat more area than the actual $5 \mathrm{G}$ contour. We have connected several regions of $5 \mathrm{G}$ magnetic field together when in fact they are separated by an area of lower field. This is probably because the borders where the field drops off more rapidly to low values are easier to define than are the $5 \mathrm{G}$ contours in a region where the field is only changing gradually from 6 to $4 \mathrm{G}$ and back again.

The major discrepancy between our prediction of the $5 \mathrm{G}$ contours and the actual ones mapped by Mt. Wilson is the neutral line between the plages and the spot group. The actual line is more curved than predicted in our sketch so that an arm of following polarity curves around to the south of the spot group.

\section{Identifications of Magnetic Features of January 2, 1969}

Figure 4 shows an exceptionally fine $\mathrm{H} \alpha+0.5 \AA$ picture of two mature (second observed rotation) active regions on January 2, 1969, with an overlay of longitudinal magnetic field contours from the daily Mt. Wilson magnetogram. A $\frac{1}{4} \AA$ Zeiss filter was used this day and the seeing was excellent. The disturbed chromospheric region is clearly visible. The $5 \mathrm{G}$ contour outlines the network of dark, enhanced ridges of spicules.* The existence of 'herringbone' spicule* structures has been pointed out by

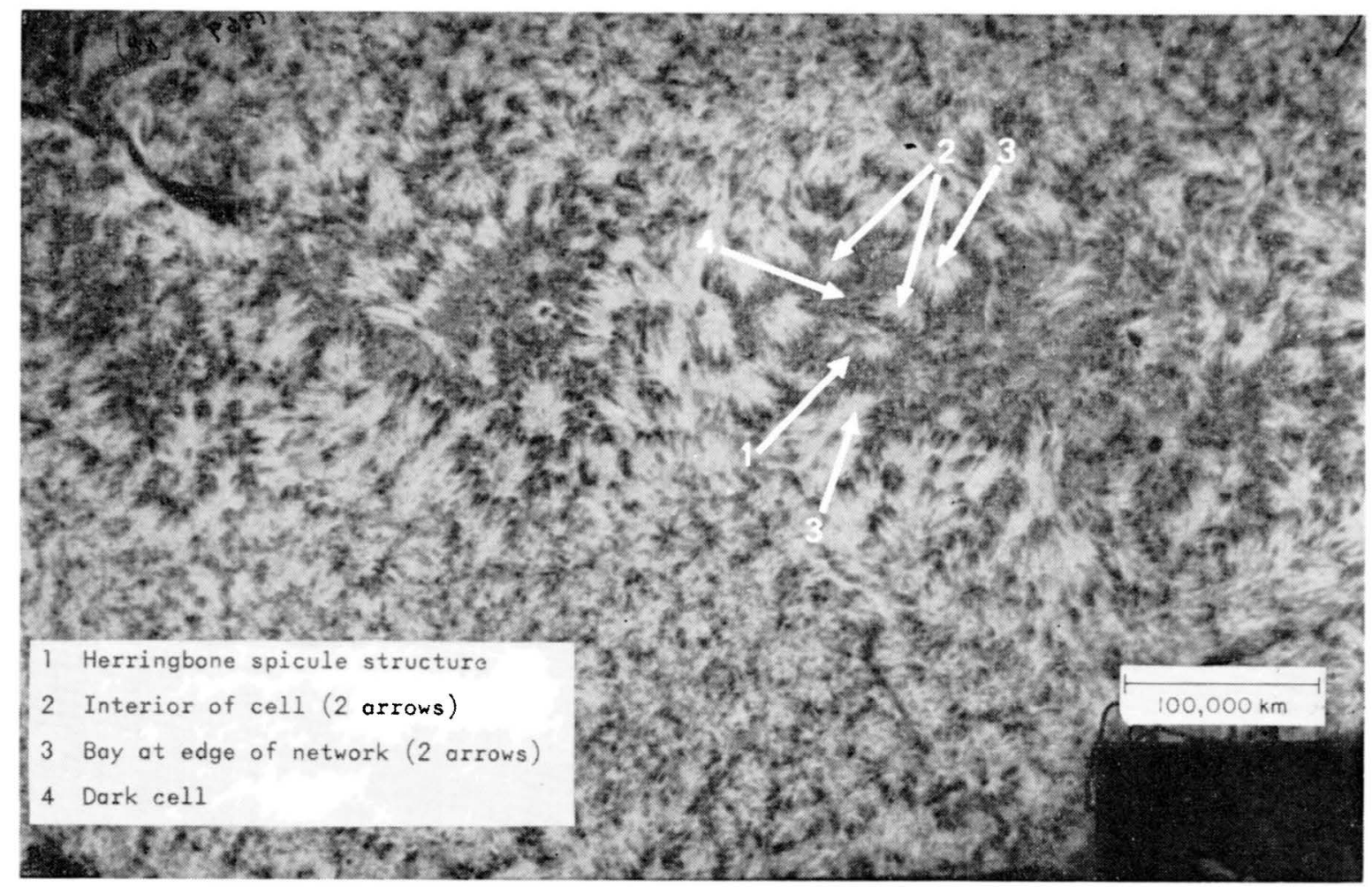

Fig. 4. Two bipolar groups January 2, $1969 \mathrm{H} \alpha+\frac{1}{2} \AA$.

* The identification of these spikes as limb spicules is not yet fully established, but their appearance on the disk is similar. 
veasons indicated. In general, we have indicated the regions of magnetic fields stronger than $5 \mathrm{G}$ and have assigned the correct polarities to them. On a smaller scale, our contour tends to include somewhat more area than the actual $5 \mathrm{G}$ contour. We have connected several regions of $5 \mathrm{G}$ magnetic field together when in fact they are separated by an area of lower field. This is probably because the borders where the field drops off more rapidly to low values are easier to define than are the $5 \mathrm{G}$ contours in a region where the field is only changing gradually from 6 to $4 \mathrm{G}$ and back again.

The major discrepancy between our prediction of the $5 \mathrm{G}$ contours and the actual ones mapped by Mt. Wilson is the neutral line between the plages and the spot group. The actual line is more curved than predicted in our sketch so that an arm of following polarity curves around to the south of the spot group.

\section{Identifications of Magnetic Features of January 2, 1969}

Figure 4 shows an exceptionally fine $\mathrm{H} \alpha+0.5 \AA$ picture of two mature (second observed rotation) active regions on January 2,1969 , with an overlay of longitudinal magnetic field contours from the daily Mt. Wilson magnetogram. A $\frac{1}{4} \AA$ Zeiss filter was used this day and the seeing was excellent. The disturbed chromospheric region is clearly visible. The $5 \mathrm{G}$ contour outlines the network of dark, enhanced ridges of spicules.* The existence of 'herringbone' spicule* structures has been pointed out by

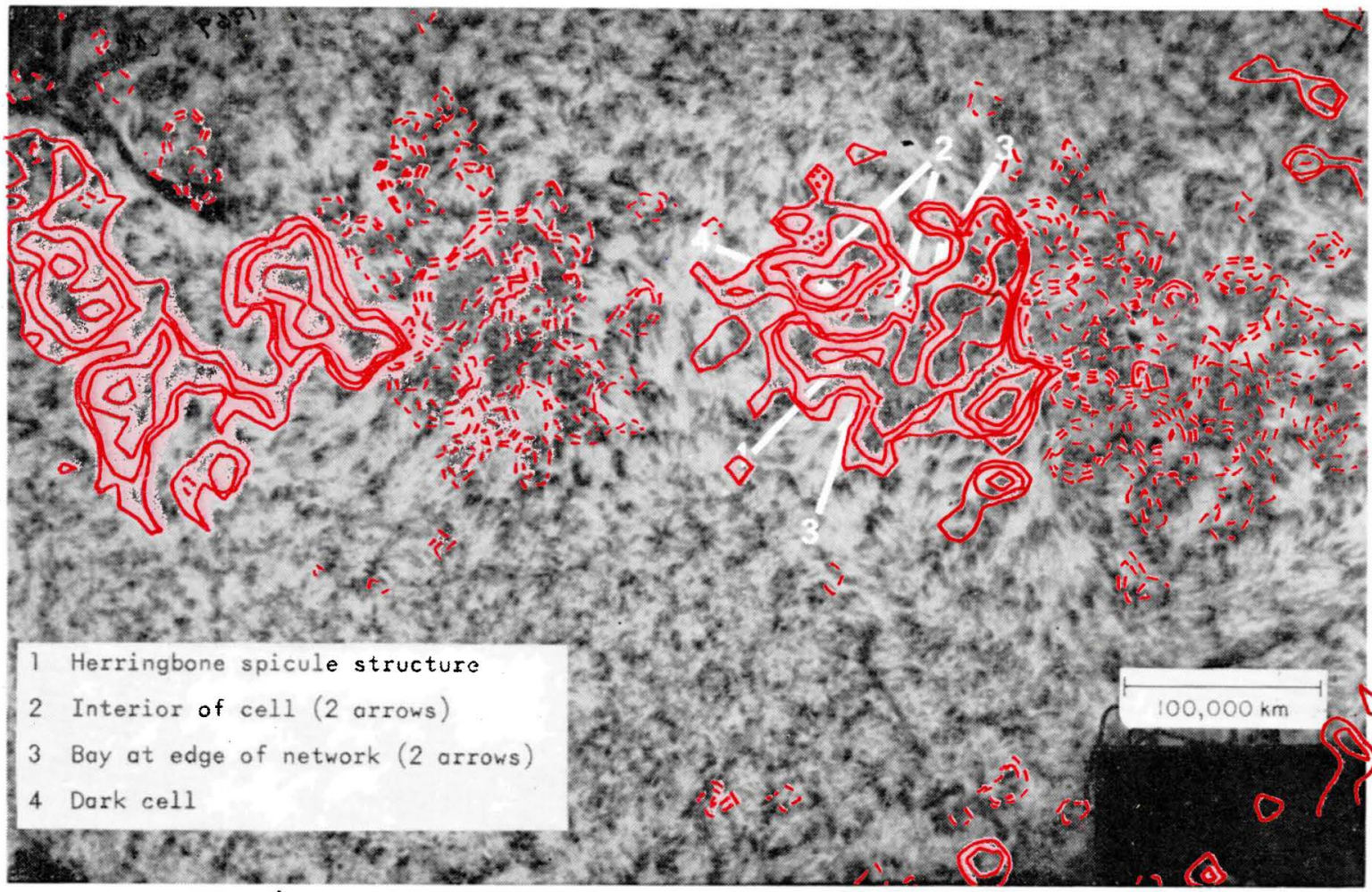

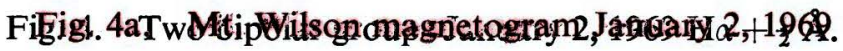

* The identification of these spikes as limb spicules is not yet fully established, but their appearance on the disk is similar. 
Beckers $(1963,1968)$ and by Howard and Harvey. There are several examples of this type of structure evident in Figure 4 (arrow No. 1) and they seem to coincide with narrow, elongated regions of higher magnetic field. However, the more typical spicule ridge is only a single row. The single rows and 'herringbones' form a network of ridges in the $\mathrm{H} \alpha+0.5 \AA$ picture. A single cell consists of a light or dark interior surrounded by a ridge of spicules.

Whenever the light interior of a cell in an off-band picture (i.e. a narrower absorption line profile) is large enough to be resolved by the magnetograph, it shows as a field minimum (see Figure 4, arrows No. 2). Unfortunately, most of these cells are not resolved (i.e. size less than $17^{\prime \prime}$ or field difference less than $5 \mathrm{G}$ ). In several cases, ridges of spicules on the edge of the enhanced region form a cell which is incomplete. The resulting 'bay' is very similar to completely enclosed cells except that it is missing one spicule ridge border (see Figure 4, arrows No. 3). There is a continuous transition from bays to cells. The bays are typically resolved in the magnetograms as regions with fields lower than $5 \mathrm{G}$. The ridges bordering them show fields greater than $5 \mathrm{G}$. We would guess that the light areas within the $5 \mathrm{G}$ contour (arrows No. 2) always correspond to vertical field values below $5 \mathrm{G}$.

Regions of greater magnetic fields $(\sim 20 \mathrm{G})$ are characterized by filled-in cells (i.e. dark in $\mathrm{H} \alpha+0.5 \AA$ ) and eventually (in the center of $\mathrm{H} \alpha$ ) by plages. The work of Sheeley (1965) and others shows that the small scale fields are more intense than the nominal magnetograph contours would indicate. The field intensities in the ridges are probably of the order of $100 \mathrm{G}$ with very little field between ridges, but without high resolution magnetograms we cannot prove this.

Leighton (1959) mentioned that the magnetic fields around active regions (outside of the penumbra) had filamentary structure, but he did not identify this magnetic structure with any visible structure. We submit that this filamentary magnetic structure would coincide with the network of spicule ridges around the active regions. Since the $5 \mathrm{G}$ contour established the boundary of unipolar regions in the work of Bumba and Howard (1966), our regions are obviously the same thing.

There is an extensive region of disturbed chromospheric structure between the $5 \mathrm{G}$ magnetic contour (or the limit of the spicule ridges) and the line of zero vertical field marked by prominences. This region shows fibril structures and less violent oscillatory motion than outside it. This disturbed region surrounds the outer $5 \mathrm{G}$ contour and is distinct from both the spicule network and the undisturbed chromosphere. Since magnetic observations are not available for fields below $5 \mathrm{G}$, and we have no transverse field data, we cannot give an ironclad rule for determining the field strength in these regions. But it appears highly likely that the field there is predominantly horizontal and fairly strong ( $\gtrsim 5 \mathrm{G})$.

The disturbed region of horizontal field is most evident where the field changes sign between two extensivi unipolar regions. (Cf. the center section of Figure 4 between the two bipolar groups.) It seems logical that the field does not decrease in intensity here, but simply changes sign. However, in almost all cases the surface fibrils are parallel to the boundary, rather then perpendicular to it so that the field lines must be 
twisted. The fibrils in the region of horizontal field are uniformly distributed and not concentrated in ridges.

The distinction between the disturbed region surrounding the $5 \mathrm{G}$ contour and the undisturbed chromosphere still further away from the active region is best seen in the motion pictures. The oscillation within this disturbed region is distinctly less violent than the oscillation in the undisturbed chromosphere. There are no dark spicules in this region so that it appears slightly lighter than the undisturbed chromosphere. It is amusing that the 'quiet' chromosphere is best distinguished in films by the presence of violent oscillations.

The network of spicule ridges around the active region within the $5 \mathrm{G}$ magnetic contour possesses considerable fine structure. The ridges tend to outline a system of cells whose dimensions are typically a few times $10^{4} \mathrm{~km}$. This is comparable to the size of supergranulation cells in the quiet chromosphere as described by Simon and Leighton (1964) and others. The cells within the network of spicule ridges may be an indication of 'active supergranulation' (i.e. supergranulation modified by a general magnetic field larger than $5 \mathrm{G}$ ). Discussion of chromospheric structure should be careful to distinguish this enhanced network from the quiet chromosphere.

The cells within the enhanced network may be divided into three classes: (1) Bays at the edge of the $5 \mathrm{G}$ contour where two arms of spicules do not quite enclose a disturbed region of lower field. Bays are light, free of enhanced spicules, and are connected to the disturbed region of horizontal field outside the $5 \mathrm{G}$ contour (of longitudinal field) (see Figure 4, arrows No. 3). (2) Simple cells completely surrounded by ridges of spicules. The interiors of these cells are also light and free of enhanced spicules (see Figure 4, arrow No. 2). (3) Dark cells. These cells appear to be filled in with dark features and correspond to regions of higher magnetic field in the magnetogram (see Figure 4, arrow No. 4). Bright plages in the centerline of $\mathrm{H} \alpha$ tend to coincide with these cells that are filled in, but some filled-in cells do not correspond to plages in the centerline $\mathrm{H} \alpha$ picture (Figure 6, arrow No. 2). This would indicate that a filled-in cell is necessary but not sufficient for a plage. Roughly $20-40 \mathrm{G}$ (averaged over the $17^{\prime \prime}$ aperture of the magnetograph) seems to result in a grey cell, whereas $40 \mathrm{G}$ or more may result in a cell almost as dark as the ridges of enhanced spicules that outline it. Of course, cells of preceding polarity belong to the 'antiplage' and do not show bright in centerline $\mathrm{H} \alpha$.

The network is better defined in some parts of the disturbed region than in others. We would guess that a complicated collection of plages, or the neutral line between two regions of opposite polarity in the active region, or a region of high field gradients tend to interfere with the network. The degree of spicule enhancement is also probably dependent on the size of a region, how well connected it is, and whether it has preceding or following polarity.

The time scale of the network of spicule ridges seems to be comparable with the time scale of the magnetic field itself. During a 25 -min period of the best seeing on January 2, 1969, the network was not observed to change at all. That is, all ridges, cells, and other large features could be found in the same place throughout this 
period. Individual spicules, of course, disappeared and were replaced by new ones during the $25 \mathrm{~min}$. Examination of pictures taken on the previous day, January 1, 1969 , show that although the network has changed in its finer details over 24 hours (see Figure 5), it is still easy to pick out prominent features such as bays. The $5 \mathrm{G}$ contour seems to include about the same area on both days and its overall shape remained the same. During the previous rotation we observed a spot followed by a bright active plage which evolved into the configuration of January 1 and 2, 1969, but the disturbed region is no longer recognizable. Both bipolar groups seem to have broken up and disappeared by the next rotation.

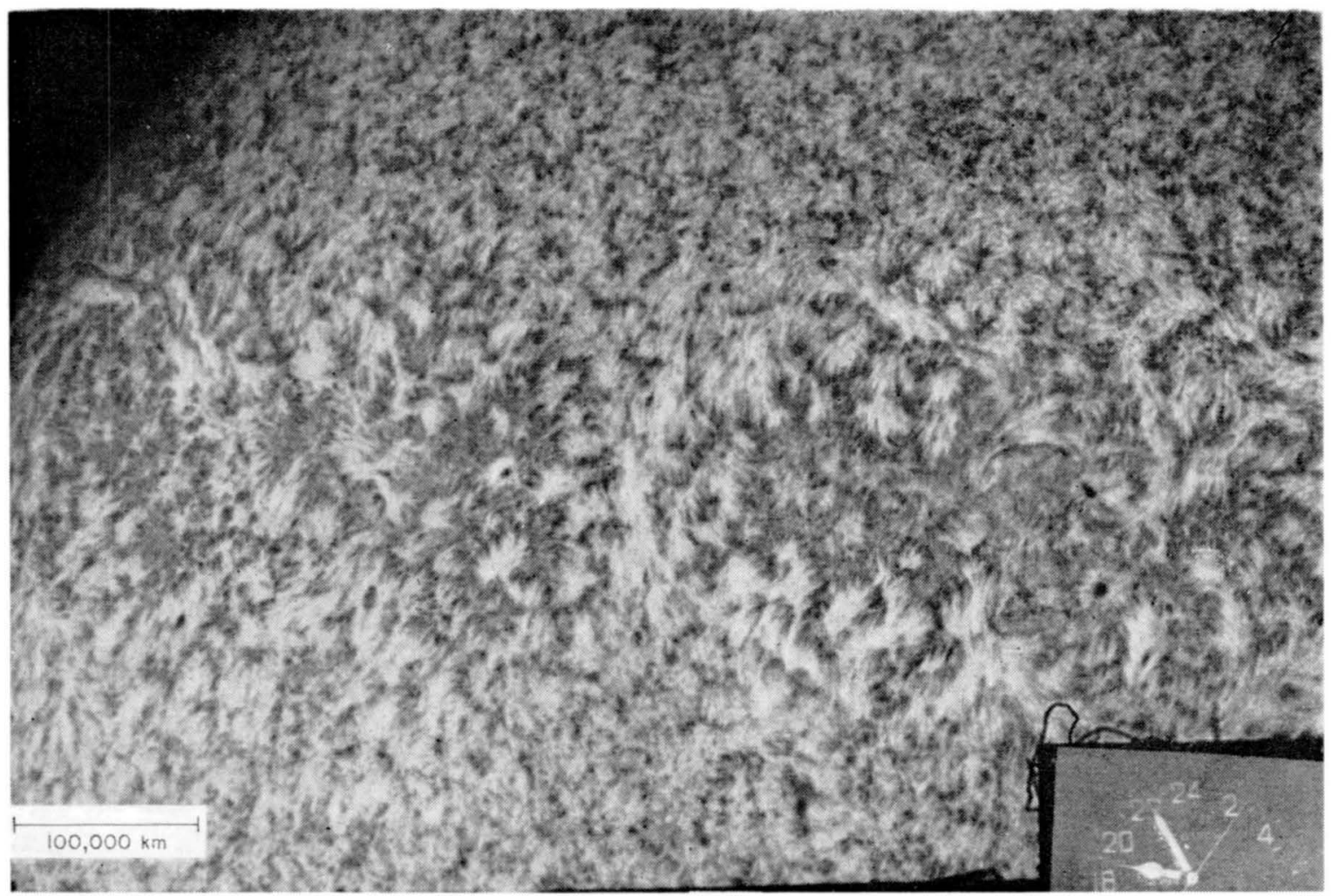

Fig. 5. Two bipolar groups January $1,1969, \mathrm{H} \alpha+\frac{1}{2} \AA$.

\section{Examples of Complicated Magnetic Configurations}

Figures 6, 7 and 8 show several complicated regions that occurred in May, 1969. In the southwest (arrow No. 1) there is a growing bipolar region on the 21 st that shows dark loops superimposed on an extended very bright plage. This is a typical 'BRL'bright region with loops (see Weart and Zirin, 1969). In Figure 7 (again arrow No. 1) the spicule structure in this region is very confused. By the 23rd (see Figure 8, arrow No. 1) this region has developed into a pair of spots and the spicule structure around them has become more stable. In such young growing regions there is not yet a welldeveloped surrounding unipolar region, but the field lines in close presumably are 
[period. Individual spicules, of course, disappeared and were replaced by new ones] during the $25 \mathrm{~min}$. Examination of pictures taken on the previous day, January 1, 1969 , show that although the network has changed in its finer details over 24 hours (see Figure 5), it is still easy to pick out prominent features such as bays. The $5 \mathrm{G}$ contour seems to include about the same area on both days and its overall shape remained the same. During the previous rotation we observed a spot followed by a bright active plage which evolved into the configuration of January 1 and 2, 1969, but the disturbed region is no longer recognizable. Both bipolar groups seem to have broken up and disappeared by the next rotation.

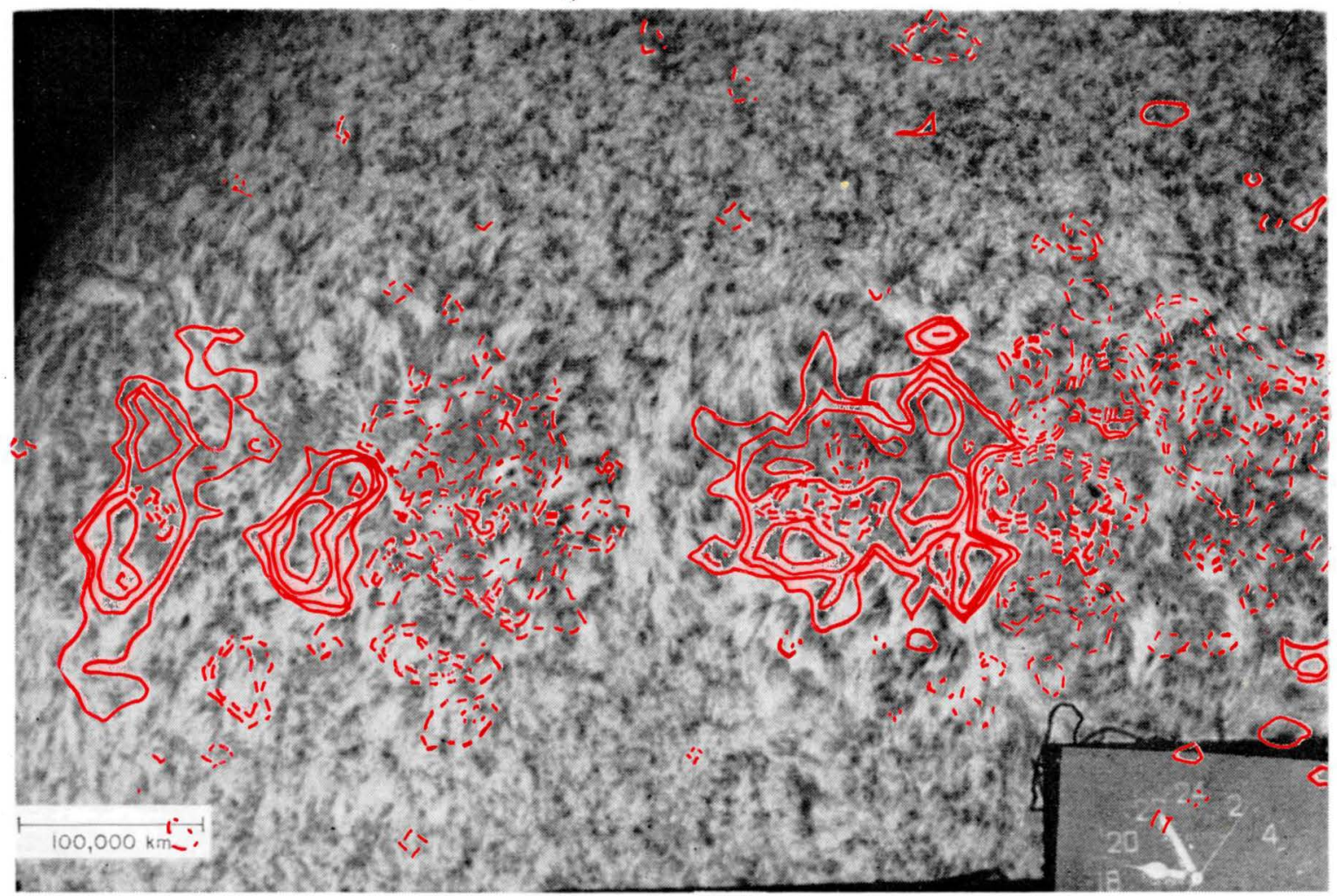



\section{Examples of Complicated Magnetic Configurations}

Figures 6, 7 and 8 show several complicated regions that occurred in May, 1969. In the southwest (arrow No, 1) there is a growing bipolar region on the 21st that shows dark loops superimposed on an extended very bright plage. This is a typical 'BRL'bright region with loops (see Weart and Zirin, 1969). In Figure 7 (again arrow No. 1) the spicule structure in this region is very confused. By the 23rd (see Figure 8, arrow No. 1) this region has developed into a pair of spots and the spicule structure around them has become more stable. In such young growing regions there is not yet a welldeveloped surrounding unipolar region, but the field lines in close presumably are 
parallel to the fibrils. Directly adjacent to the south of this region is a new growing bipolar group (arrow No. 1a) which was well developed by the 23rd. Although this side-by-side growth of two groups is unusual, there was no particular flare activity. Arrow No. 2 on these figures indicates a region of enhanced dark structure of following polarity in a bipolar group. Only part of this area has bright plage in centerline $\mathrm{H} \alpha$, but all shows some emission. Arrow No. 3 indicates the 'antiplage', i.e. a group of enhanced spicules of the preceding polarity that indicates a high magnetic field without any brightening in the centerline of $\mathrm{H} \alpha$. Arrow No. 4 indicates a very active group that had many large flares. The structure is so complex that a $5 \mathrm{G}$ contour cannot be



Fig. 6. Several complex regions May 21, 1969, H $\alpha$.

constructed from $\mathrm{H} \alpha$ pictures, but the centerline picture shows a most important magnetic characteristic. When a very bright plage of the following polarity (we recognize it as following polarity because it is bright in $\mathrm{H} \alpha$ ) curls around the preceding spot so that it intrudes into the area that is normally occupied by preceding polarity, the region will be very active and will more than likely have several large flares as long as the intrusion persists. This is particularly true if the neutral line is very close to the spot. Arrow No. 5 indicates a small growing bipolar region. 


\section{Conclusions}

The fine quality off-band picture of January 2, 1969, shows that the boundary of the network of enhanced spicules in an uncomplicated active region coincides with the $5 \mathrm{G}$ contour of longitudinal magnetic field. The magnetic field appears to share the cellular structure of the network. The spicules of following polarity are often darker and more distinct than those of preceding polarity. This asymmetry is stronger in the center of $\mathrm{H} \alpha$, where only following polarity shows bright plage (except in the case of bright regions with loops characteristic of emerging magnetic fields).

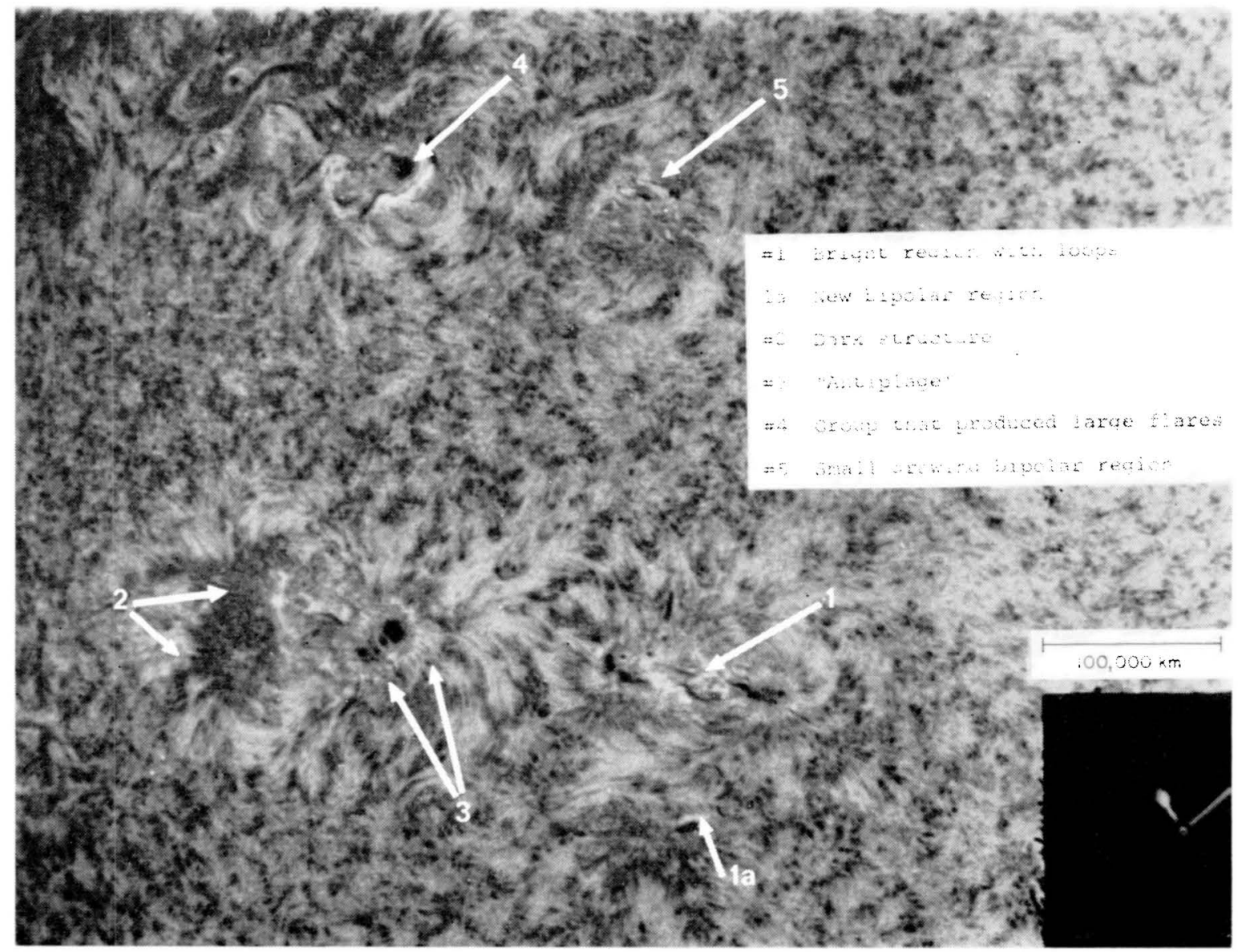

Fig. 7. Several complex regions May 21, 1969, H $\alpha+\frac{1}{2} \AA$.

Each unipolar region is surrounded by an extensive area of disturbed chromosphere in which the fibril structure indicates that the magnetic field is horizontal. It appears that there are no spicules in this region, and further, the field does not bunch up into strong clusters such as those of the network. It would be most interesting to study the limb passage of such a region, since the chromosphere-corona transition zone must have an unusual nature there.

It is clear from this work that we must recognize three distinct regions of the 
chromosphere: the enhanced network within the $5 \mathrm{G}$ contour (made up of elements of much larger magnetic field interspersed with horizontal field), the disturbed region of horizontal (transverse) field, and the undisturbed, but oscillating, quiet chromosphere.

Our results show that, given a few sample magnetograms from which to learn the rules, we may easily determine the magnetic field structure in the chromosphere. In the case of transverse fields, it would appear possible to improve on magnetograph results by inference. Of course, the success of the chromospheric magnetograph is due to the great regularities of chromospheric structure, in which only certain welldefined magnetic configurations occur.

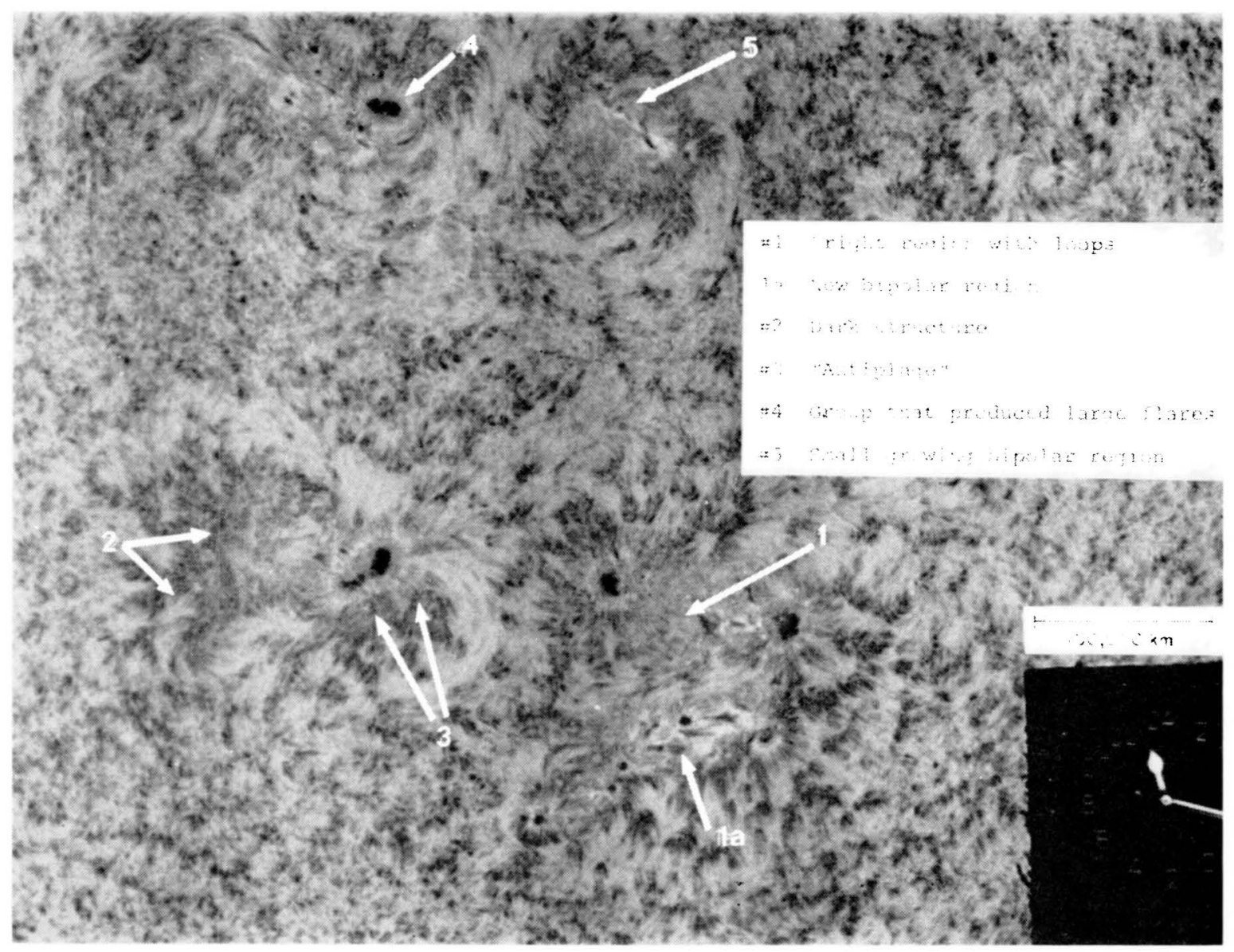

Fig. 8. Several complex regions May 23, 1969, H $\alpha+\frac{1}{2} \AA$.

\section{Acknowledgement}

We would like to thank NSF for the traineeship of Glenn J. Veeder. This work has been supported by NASA Contract No. NGR 05-002-034 and NSF under Grant No. 1472. 


\section{References}

Beckers, J. M.: 1963, Astrophys. J. 138, 648.

Beckers, J. M.: 1968, Solar Phys. 3, 367.

Bumba, V. and Howard, R.: 1966, Astrophys. J. 143, 592.

Howard, R. and Harvey, J. W.: 1964, Astrophys. J. 139, 1328.

Leighton, R. B.: 1959, Astrophys. J. 130, 366.

Sheeley, N. R.: 1965, Measurement of Solar Magnetic Fields, Caltech Thesis.

Simon, G. W. and Leighton, R. B.: 1964, Astrophys. J. 140, 1120.

Weart, S. R. and Zirin, H.: 1969, Publ. Astron. Soc. Pacific 81, 270. 